\title{
Treatment of advanced pancreatic neuroendocrine tumors: potential role of everolimus
}

This article was published in the following Dove Press journal:

OncoTargets and Therapy

4 April 2012

Number of times this article has been viewed

\section{Putao Cen \\ Robert J Amato}

Division of Oncology, Department of Internal Medicine, University of Texas Health Science Center at Houston (Medical School), Houston, TX, USA
Correspondence: Robert J Amato The University of Texas, Memorial Hermann Cancer Center, 6410 Fannin St, Suite 830 , Houston, TX 77030, USA Tel + I 8323257702

Email robert.amato@uth.tmc.edu
Abstract: Pancreatic neuroendocrine tumors (PanNETs) are frequently diagnosed at unresectable stage and remain a medical challenge. Everolimus (RAD001, Afinitor ${ }^{\circledR}$, Novartis, Basel, Switzerland), an orally administered inhibitor of mammalian target of rapamycin (mTOR), was recently approved by the Food and Drug Administration to treat patients with advanced PanNETs. This review will examine the mechanism of action of everolimus, the function of mTOR and its inhibition, PanNETs and the mTOR pathway, and clinical trials of everolimus in PanNETs. Future investigations will focus on everolimus combination therapy to treat PanNETs and the discovery of predictive biomarkers for response to everolimus.

Keywords: pancreatic neuroendocrine tumor, PanNETs, everolimus, RAD001, mTOR inhibitor, biomarker

\section{Introduction}

Pancreatic neuroendocrine tumors (PanNETs), also called islet cell tumors, are the second-most common malignancy of the pancreas. From population-based studies, PanNETs represent approximately $1.3 \%$ of all cases of pancreatic cancer in incidence and $10 \%$ of cases in prevalence. ${ }^{1,2}$ They are frequently diagnosed at a late stage. From the Surveillance, Epidemiology, and End Results (SEER) database, patients who presented with distant metastatic disease had a median survival of 2 years. ${ }^{2}$ Around half of PanNETs are nonfunctional, and 90\% of these are malignant. Of those functioning tumors (except insulinoma), the majority are malignant with metastatic potential and include glucagonomas, gastrinomas, somatostatinomas, VIPoma, and pancreatic polypeptidoma.

For patients with stage 4 limited hepatic diseases, surgical excision of both the liver metastases and primary pancreatic lesion should be considered. For those with unresectable disease who are asymptomatic with low tumor burden and stable disease, monitoring closely with hormone markers and imaging is recommended. For patients with unresectable metastatic disease who have significant tumor burden, clinical progression, or disease-related symptoms, treatment options include hepatic regional direct therapies, systemic cytotoxic chemotherapy, somatostatin analogs, peptide receptor radionuclide therapy (PRRT), and, most recently, targeted therapies. Somatostatin analogues relieve hormone-related symptoms in functioning tumors. A recent study of mid-gut carcinoid tumors ${ }^{3}$ found that somatostatin analogs may possibly delay disease progression in PanNETs; however, it needs to be confirmed in future randomized clinical trials of PanNETs. Systemic cytotoxic chemotherapies are 
mainly applied from studies of streptozocin-based regimens $s^{4,5}$ and temozolomide-based regimens. ${ }^{6-9}$ The partial response (PR) rates according to Response Evaluation Criteria In Solid Tumors (RECIST) criteria from these regimens were reported with range from $10 \%-70 \%$. However, these cytotoxic chemotherapy regimens were associated with considerable adverse events in non-curable patients. PRRT is an investigational therapy that uses somatostatin analogs labeled with radionuclides (yttrium-90 or lutetium-177) to deliver higher radiation doses targeted to the neuroendocrine tumor. Renal toxicity can occur from this therapy, and randomized clinical trials are needed to confirm its benefit over other agents.

Recent studies showed several growth factor receptor pathways are involved in PanNETs, especially, the mammalian target of rapamycin (mTOR) pathway. ${ }^{10-12}$ The mTOR is a molecule that controls a variety of signaling pathways that regulate many facets of cell growth and metabolism. Rapamycin (sirolimus), a macrolide antibiotic and antifungal agent, was discovered in 1975. Because of its immunosuppressant and growth inhibitory properties, it has been used clinically to prevent graft rejection following organ transplants. The rapamycin molecule has been modified to improve pharmacologic characteristics to optimally exploit its role as an anticancer agent. These rapamycin analogs are known as rapalogs. Everolimus (Afinitor ${ }^{\circledR}$, Novartis, Basel, Switzerland) is the 40-O-(2-hydroxyethyl) derivative of rapamycin. In May 2011, everolimus was approved by the Food and Drug Administration (FDA) for the treatment of progressive PanNETs, based on the results of a phase III randomized clinical trial. ${ }^{13}$

\section{The mammalian target of rapamycin (mTOR)}

The mTOR molecule is a $290 \mathrm{kD}$ serine-threonine protein kinase that acts as a biologic switch. It is central in controlling cellular responses to alterations in the microenvironment and nutrient status by influencing protein translation and gene transcription as well as governing progression in the cell cycle from G1 to S. ${ }^{14}$

The molecule's activity is affected by a variety of factors that influence cell functioning (Figure 1). Information transferred via receptors and multiple signaling molecules permits mTOR to sense the levels of nutrients available for cell growth, including glucose, amino acids, and energy. The mTOR molecule responds to external signals such as cytokines, hormones, and growth factors, and also reacts to cellular stresses such as hypoxia, $\mathrm{pH}$ or osmotic alterations, heat shock, oxidative stress, and DNA damage. The pathways

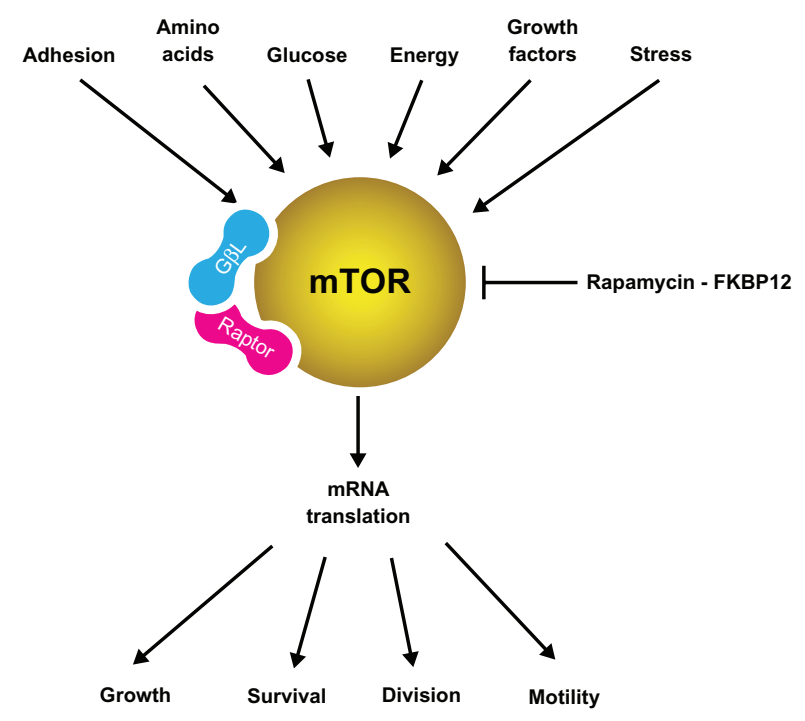

Figure I mTOR controls responses to changes in the cellular environment. Abbreviations: mTOR, mammalian target of rapamycin; mRNA, messenger ribonucleic acid.

by which signals are transduced to mTOR are complex and include some of the most critical molecules known to be dysregulated in cancer. Figure 2 represents some of the molecules that regulate mTOR activation. When the two components of the tuberous sclerosis complex (TSC, comprised of TSC1 [hamartin] and TSC2 [tuberin]) heterodimerize, mTOR is inhibited and can no longer phosphorylate downstream substrates. ${ }^{15}$ The TSC intermediate filaments (iFs) are regulated, in part, by the growth-stimulating kinase Akt. Phosphorylation of TSC2 by Akt promotes dissociation of the TSC1/TSC2 complex, which activates the guanosine

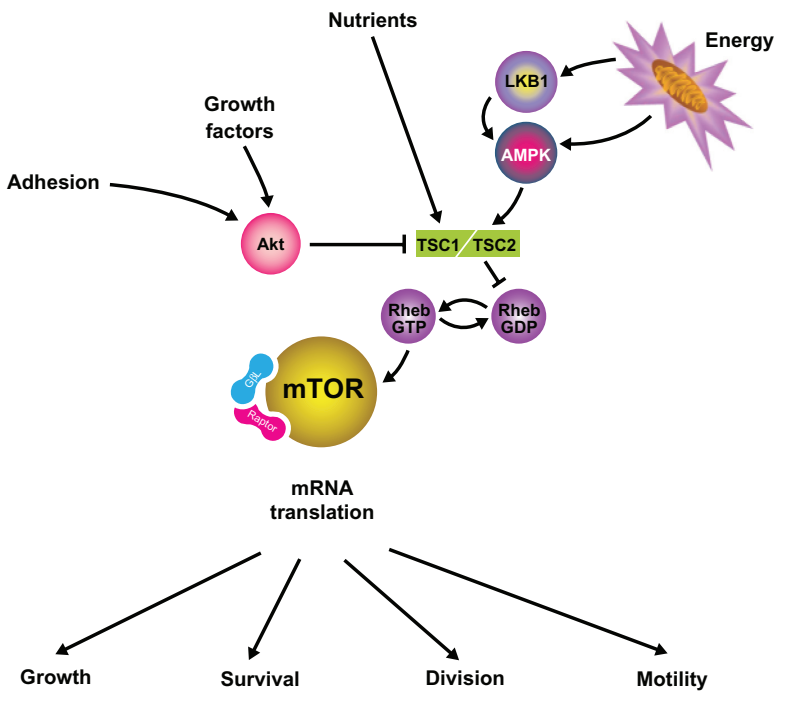

Figure 2 mTOR activation is controlled by the TSCI/TSC2 complex and Rheb. Abbreviations: Rheb GTP, ras homolog enriched in brain Guanosine- $5^{\prime}$-triphosphate; LKBI, liver kinase BI; AMPK, adenosine monophosphate kinase; Akt, also known as protein kinase $B$ (PKB); Rheb GTP, ras homolog enriched in brain guanosine diphosphate. 
triphosphatase Rheb; Rheb activity subsequently upregulates mTOR, stimulating its activity to promote messenger mRNA translation. TSC responds to a variety of other signals, including amino acid levels or energy levels. Low cellular energy levels, as measured by the adenosine triphosphate:adenosine monophosphate (ATP:AMP) ratio, activate the kinase AMPK, phosphorylating and activation TSC2, promoting its association with TSC1 and thereby inactivating Rheb, leading to mTOR inhibition. These scenarios demonstrate how signals transduced through the TSC are mediated by Rheb to affect mTOR, although the precise interactions between Rheb and mTOR have not yet been entirely elucidated. At least two other regulatory molecules have been shown to affect mTOR activity, including raptor (regulatory-associated protein of mTOR) and G $\beta L$.

Figure 3 illustrates how mTOR allows cells to respond quickly to stress by affecting both protein translation as well as gene transcription. S6 kinase 1 (S6K1) is an enzyme whose activation by mTOR results in the phosphorylation of ribosomal protein $\mathrm{S} 6$, stimulating the translation of $5^{\prime}$-terminal oligo-pyrimide (TOP) mRNAs. ${ }^{16}$ TOP mRNAs are responsible for production of key components permitting protein translation, including elongation factors and poly- $\mathrm{A}$ binding protein; ribosome production is dependent upon translation of TOP mRNAs.

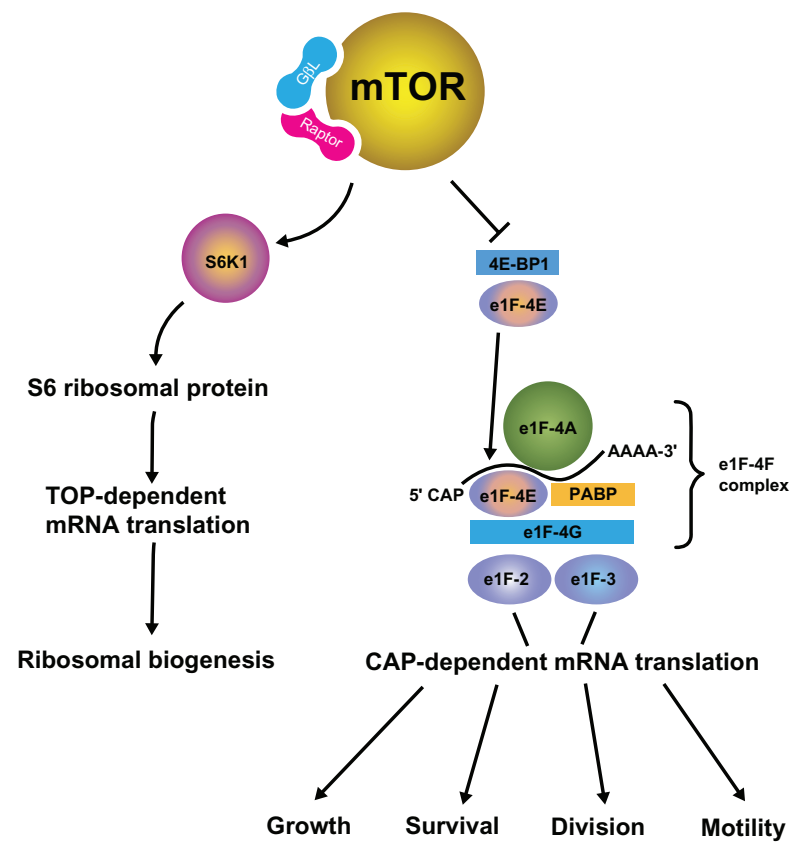

Figure $3 \mathrm{mTOR}$ controls ribosome biogenesis and CAP-dependent mRNA translation. Abbreviations: mTOR, mammalian target of rapamycin; S6KI, S6 kinase beta-I; mRNA, messenger ribonucleic acid; TOP, polypyrimidine tract; $4 \mathrm{E}-\mathrm{BPI}$, 4E-binding protein I; elF-4E, elongation factor 4E; elF-4A, elongation factor 4A; elf-4F, elongation factor 4F; elF-4G, elongation factor 4G; elF-2, elongation factor 2; elF-3, elongation factor 3; PABP, poly A binding protein; CAP, catabolic activator protein; $\mathrm{G} \beta \mathrm{L}, \mathrm{G}$ protein $\beta$-like.
Cap-dependent mRNA translation is controlled by mTOR through the regulation of elongation factor $4 \mathrm{E}$ (elF-4E) with 4E-binding protein 1 (4E-BP1). Release of elF-4E from its binding protein permits its association with other initiation and elongation factors to form active elF-4F complexes. These complexes recognize Cap-mRNAs, which code for important proteins that regulate the cell cycle, cell growth, and susceptibility of cells to apoptosis (programmed cell death). Activation of mTOR promotes Cap-mRNA translation, and mTOR inhibition rapidly shuts this off by increasing association of elF-4E with its binding protein, limiting its availability to form active elF-4F complexes. Altering the translation of some of these key proteins suggests an attractive strategy through which mTOR inhibition could potentially impede cancer progression. ${ }^{17}$

Hypoxia-inducible factor $1 \alpha(\mathrm{HIF}-1 \alpha)$ is among the important proteins encoded by capped mRNAs. HIF-1 $\alpha$ plays a key role in regulating the transcription of the vascular endothelial growth factor (VEGF) gene, which is known to be a potent stimulator of angiogenesis in cancer. Overexpression of HIF-1 $\alpha$ correlates with aggressive disease and poor prognosis in numerous tumor types; often, these are cancers known to possess signaling defects in mTOR-related pathways. ${ }^{18}$ Figure 4 illustrates the relationship between mTOR, angiogenesis, and responses to hypoxic stress. When the oxygen supply

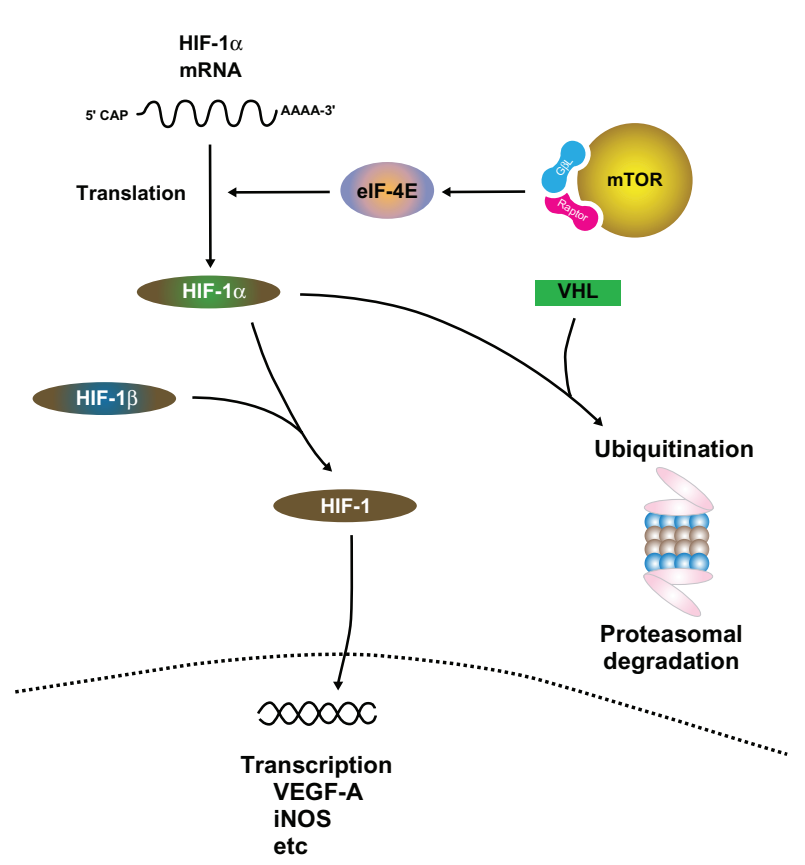

Figure 4 mTOR controls responses to hypoxic stress.

Abbreviations: mTOR, mammalian target of rapamycin; mRNA, messenger ribonucleic acid; VHL, Von Hippel-Lindau; HIF-I $\alpha$, hypoxia-inducible factors I alpha; HIF-I $\beta$, hypoxia-inducible factors I beta; HIF-I, hypoxia-inducible factors I;VEGF-A, vascular endothelial growth factor $A$; iNOS, inducible nitric oxide synthase; $G \beta L$, G protein $\beta$-like. 
is sufficient, HIF- $1 \alpha$ is continuously produced and rapidly degraded via interaction with the Von Hippel-Lindau (VHL) protein, which facilitates ubiquitination and subsequent proteasomal degradation. Under hypoxic conditions, HIF- $1 \alpha$ is not degraded; instead, it is translocated to the nucleus where it joins with HIF-1 $\beta$ to stimulate the initiation of transcription of an array of hypoxia-regulated genes, including VEGF. In rapidly progressing disease, tumor mass often outstrips blood supply, and tumor cells become hypoxic. The HIF response to hypoxia effectively promotes angiogenesis and enhances tumor survival. ${ }^{19}$ Interestingly, mTOR regulates expression of hypoxia-inducible genes. Importantly, the VHL protein is lost in the clear cell subtype of renal cell carcinoma (RCC), providing a concrete rationale for considering antiangiogenic agents for its treatment.

Carcinogenesis is a result of dysregulation of cell proliferation or apoptosis at a variety of levels. Figure 5 illustrates the complexity of many of the signaling pathways that ultimately impact gene transcription. It is evident that a substantial number of these act through mTOR, highlighting its role as a central cellular biologic switch. Disruption of inappropriate signals to influence cell proliferation at a convergent point, such as the mTOR molecule, might represent a viable and efficient approach to cancer treatment. A variety

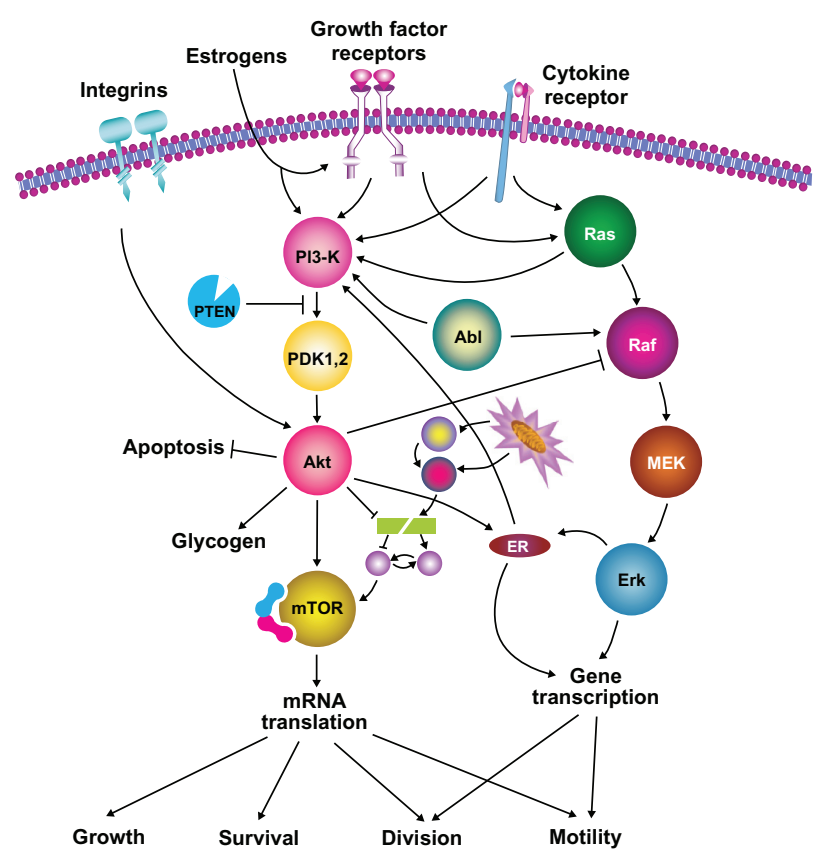

Figure 5 Growth factor signaling pathways are interconnected.

Abbreviations: mTOR, mammalian target of rapamycin; mRNA, messenger ribonucleic acid; PI3-K, phosphoinositide 3-kinase inhibitor; PDKI,2, phosphoinositide-dependent kinase-I; Abl, Abelson gene; Akt, protein kinase Akt; ER, estrogen receptor; Erk, extracellular-signal-regulated kinases; Ras, RAt sarcoma kinase; Raf, rapidly accelerated fibrosarcoma kinase; MEK, mitogen-activated protein kinase; PTEN, phosphatase and tensin homolog. of common tumor types, including lung, breast, colon, and head and neck cancers, are characterized by the overexpression of growth factor receptors or the overproduction of the growth factors themselves, which results in the inappropriate downstream stimulation of cell growth. Complex cascades of signaling enzymes mediate cellular processes that affect gene transcription; the redundancy within this system provides for exquisite sensitivity to respond to environmental changes when the system is operating properly, while also permitting numerous points for aberrant signaling to promote oncogenesis.

The Ras-Raf-MEK pathway and the phosphoinositide-3 kinase (P13K)-Akt pathway are the key regulators of cell proliferation. Oncogenic mutations in RAS are common in many tumor types, including pancreas, colon, thyroid, lung cancers, and myeloid leukemias. ${ }^{20,21}$ Cross-talk between cellular communication pathways also occurs. Both RAS and $\mathrm{Abl}$ kinase, for example, promote stimulatory signals through the Ras-Raf-MEK route as well as through P13K. Mutations or amplifications in the gene for the catalytic subunit of P13K have been detected in glioblastomas, gastric, colon, breast, and ovarian cancers. Akt is another important enzyme regulating mTOR; it is constitutively activated in many tumor types. ${ }^{22}$ In addition to inappropriately driving cell proliferation, constitutively active Akt may represent one mechanism by which cells escape the effects of cytotoxic chemotherapy. Genetic damage inflicted by chemotherapy drugs should activate apoptosis, but constitutively activated Akt is believed to block this. Clinical evidence confirms that high levels of constitutively active Akt in breast cancer correlate with resistance to endocrine therapy and poor prognosis. ${ }^{23}$ The phosphatase and tensin homologue (PTEN) modifies the activity of P13K. PTEN dephosphorylates activated P13K, thereby inactivating it, and preventing downstream signaling through the P13K-Akt pathway. Mutations in PTEN can result in loss of function of this molecule, thereby eliminating an important step modulating signaling through P13K-Akt. PTEN mutations have been identified in many different tumor types, including endometrial cancers, melanomas, and lymphatic cancers, as well as breast, bladder, lung, ovary, and colon cancers. ${ }^{24}$

Overall, disruption of the regulation elements previously described can promote aberrant cell growth. It is clear that the complexity and interrelatedness of these signaling pathways provide fertile ground for single or multiple defects to impart proliferative advantages or confer resistance to apoptosis, promoting carcinogenesis and providing resistance to certain anticancer therapeutics. As mTOR is a critical molecule that mediates at least one of the common pathways 
for many different signaling molecules, inhibiting its activity may represent one route toward ameliorating some of the genetic defects driving tumor growth. As such, inhibition of the key biologic switch, mTOR, is proving to be a promising strategy for anticancer therapy.

\section{mTOR and PanNETs}

PanNETs are associated with several genetic syndromes involving the mTOR signaling pathway, including tuberous sclerosis complex, neurofibromatosis (NF), von Hippel-Lindau syndrome, and multiple endocrine neoplasia type $1 .{ }^{25-27}$ Expression of TSC-2 and PTEN was found downregulated in both functioning and nonfunctioning PanNETs. ${ }^{12}$ IGF-I was shown to be a major autocrine regulator of hormone secretion and tumor growth through PI3K-mTOR pathway in human PanNETs cells line. ${ }^{28}$ One study demonstrated that $14 \%$ of PanNETs have mutations in genes in the mTOR pathway. ${ }^{11}$ This finding could potentially be used to stratify patients for treatment with mTOR inhibitors.

\section{Everolimus and $\mathbf{m T O R}$}

Everolimus is a synthetic, orally available analogue of rapamycin, and it effectively inhibits mTOR activation. In PanNET cell lines that exhibit constitutively activated $\mathrm{PI}(3)-\mathrm{K} / \mathrm{Akt} / \mathrm{mTOR}$ signaling, everolimus was shown to induce apotosis and cell growth arrest involved G0/G1

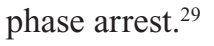

\section{Clinical trials}

\section{Everolimus ( $5 \mathrm{mg} /$ day versus $10 \mathrm{mg} /$ day) + octreotide LAR}

Octreotide, a somatostatin analog, decreases phosphatidylinositol-3-kinase/Akt signaling in some models; ${ }^{30}$ however, a recent study in carcinoid tumor cell line didn't demonstrate synergistic or additive interactions with rapamycin (Table 1). ${ }^{31}$ Along with these controversial (or contradictory) findings, clinical studies were launched in PanNETs using this combination. A phase II clinical trial enrolled 30 carcinoid tumor patients and 30 PanNET patients (prior chemotherapy allowed) (Table 1). ${ }^{32}$ Treatment consisted of everolimus $5 \mathrm{mg} /$ day (30 patients) or $10 \mathrm{mg} /$ day (30 patients) and octreotide LAR $30 \mathrm{mg}$ every 28 days. Seventeen percent of 30 carcinoid patients achieved partial response (PR), and 27\% of 30 PanNET patients achieved PR. Stratified by everolimus dose, $13 \%$ of patients achieved PR in the $5 \mathrm{mg}$ cohort, and $30 \%$ of patients achieved PRs in the $10 \mathrm{mg}$ cohort. However, there were more carcinoid patients treated at $5 \mathrm{mg}$ and more PanNETs patients treated at $10 \mathrm{mg}$. Among the 37 patients who had elevated chromogranin A level at study entry, 70\% achieved normalization or more than a $50 \%$ reduction. The chromogranin A response rates among patients who either had prior octreotide or were octreotide-naïve were $67 \%$ and $77 \%$, respectively. Progression free survival (PFS) was 63 weeks for carcinoid patients and 50 weeks for PanNET patients. By everolimus dose level, median PFS of patients treated with $5 \mathrm{mg}$ and $10 \mathrm{mg}$ of RAD001 was 50 weeks and 72 weeks, respectively. Prior octreotide therapy had no impact on median PFS, which was 60 weeks in both groups. Grade 3 thrombocytopenia occurred in $3 \%$ of the $5 \mathrm{mg}$ cohort and $6 \%$ of the $10 \mathrm{mg}$ cohort. Grade 3/4 leukopenia occurred in $3 \%$ of patients in the $5 \mathrm{mg}$ cohort and in $6 \%$ in the $10 \mathrm{mg}$ cohort. No pneumonitis was observed at the $5 \mathrm{mg}$ dose level. However, at the $10 \mathrm{mg}$ dose level, 3 patients ( $9 \%$ ) experienced grade 2 pneumonitis and one patient experienced grade 3 pneumonitis.

\section{Everolimus versus}

\section{everolimus + octreotide LAR}

RADIANT-1 (RAD001 in Advanced Neuroendocrine Tumors), a phase II study, enrolled 160 patients with advanced PanNETs and evidence of RECIST-defined progression after cytotoxic chemotherapy (Table 1$).{ }^{33}$ Patients $(n=115)$ who

Table I Everolimus clinical trials

\begin{tabular}{|c|c|c|c|c|c|}
\hline Clinical trial & Regimens & $\begin{array}{l}\text { Tumor } \\
\text { type }\end{array}$ & $\begin{array}{l}\text { Patient } \\
\text { no. }\end{array}$ & $\begin{array}{l}\text { Response } \\
\text { rate }\end{array}$ & $\begin{array}{l}\text { PFS } \\
\text { (months) }\end{array}$ \\
\hline \multirow[t]{2}{*}{ Phase $\|^{32}$} & Everolimus ( $5 \mathrm{mg} /$ day versus $10 \mathrm{mg} /$ day $)+$ & Carcinoid & 30 & $17 \%$ & 14.5 \\
\hline & octreotide LAR & PanNETs & 30 & $27 \%$ & II.5 \\
\hline \multirow[t]{2}{*}{ Phase II RADIANT- I ${ }^{33}$} & Everolimus & PanNETs & 115 & $9 \%$ & 9.7 \\
\hline & Everolimus + octreotide LAR & & 45 & $4 \%$ & 16.7 \\
\hline Phase $\|^{34}$ & Everolimus + bevacizumab & PanNETs & 39 & $8 \%$ & 14.6 \\
\hline \multirow[t]{2}{*}{ Phase III RADIANT-3'3 } & Everolimus & PanNETs & 207 & $5 \%$ & II \\
\hline & Placebo & & 203 & $2 \%$ & 4.6 \\
\hline \multirow[t]{2}{*}{ Planned phase II } & Everolimus + octreotide LAR & PanNETs & & & \\
\hline & Everolimus + octreotide LAR + Bevacizumab & & & & \\
\hline
\end{tabular}

Abbreviation: PFS, progression-free survival. 
were not being treated with octreotide at study entry were assigned to stratum 1 (everolimus $10 \mathrm{mg}$ daily orally), and patients $(n=45)$ who were on octreotide LAR for at least 3 consecutive months at study entry were assigned to stratum 2 (everolimus $10 \mathrm{mg}$ daily orally plus octreotide LAR). Patients enrolled in stratum 2 had documented disease progression while receiving octreotide LAR. In the everolimus alone cohort, $9.6 \%$ of patients received PR with a median PFS of 9.7 months and an overall survival (OS) of 24.9 months. In the everolimus plus octreotide LAR cohort, $4.4 \%$ of patients achieved PR with a median PFS of 16.7 months and OS was unreached at data cut-off. Patients with early chromogranin A or neuron-specific enolase level response had a longer PFS in comparison to patients without an early response. The most frequent grade 3 or 4 adverse events suspected to be related to study drug were asthenia in stratum 1 and thrombocytopenia in stratum 2. The adverse events that most often required dosage adjustment or interruption were hyperglycemia $(7.8 \%)$, diarrhea $(5.2 \%)$, stomatitis $(7.0 \%)$, and pyrexia $(4.3 \%)$ in stratum 1 , and thrombocytopenia $(11.1 \%)$, stomatitis $(8.9 \%)$, and pyrexia $(11.1 \%)$ in stratum 2 . The pharmacokinetics study showed coadministration of octreotide LAR and everolimus did not have clinically significant effects on the exposure of either drug by itself.

\section{Everolimus versus placebo}

In a subsequent randomized phase III study (RADIANT-3), 410 patients with advanced PanNETs and radiologic disease progression were randomized to receive everolimus (10 mg/day) or a placebo (Table 1). ${ }^{13}$ More than $90 \%$ of patients had metastases in the liver, and approximately $60 \%$ received a diagnosis of PanNETs more than 2 years before entering the study. The 2 groups were similar in respect to prior treatment of chemotherapy (50\% in both groups) and somatostatin analogue therapy (49\% in the everolimus group and $50 \%$ in the placebo group).

A significant improvement in PFS was associated with everolimus in comparison to placebo (11 months versus 4.6 months, respectively; hazard ratio, 0.35 ; $95 \% \mathrm{CI}, 0.27$ to $0.45 ; P<0.01)$. The overall tumor response rate associated with everolimus in this study was $5 \%$ compared to $2 \%$ in placebo. Patients who had been assigned to the placebo initially could then switch to open-label everolimus. Median OS was not reached at the time of this analysis, and no significant difference between the groups was observed.

Drug-related adverse events were mostly grade 1 or 2 and included stomatitis (in $64 \%$ of patients in the everolimus group versus $17 \%$ in the placebo group), rash $(49 \%$ versus $10 \%)$, diarrhea $(34 \%$ versus $10 \%)$, fatigue $(31 \%$ versus $14 \%$ ), infections ( $23 \%$ versus $6 \%$ ), pneumonitis ( $17 \%$ versus $0 \%)$, infections plus pneumonitis $(12 \%$ versus $0 \%$ ), and interstitial lung disease ( $2 \%$ versus $0 \%)$. Glucocorticoids were administered to 6 of the 7 patients with grade 3 or 4 noninfectious pneumonitis or interstitial lung disease. Grade 3 or 4 events that were more frequent with everolimus than with placebo included anemia $(6 \%$ versus $0 \%$ ), hyperglycemia ( $5 \%$ versus $2 \%$ ), stomatitis, thrombocytopenia, diarrhea, hypophosphatemia, and neutropenia. Adverse events related to the study drug led to the discontinuation of treatment in $13 \%$ of the patients receiving everolimus (with pneumonitis, fatigue, and interstitial lung disease cited as the most common reasons).

\section{Everolimus + bevacizumab}

PanNET is a hypervascular tumor. Clinical investigations using the combination of everolimus with bevacizumab to further target the vasculature of PanNETs were conducted. Everolimus combined with bevacizumab was shown an overall tumor response rate of 21\% (8/39 patients) and PFS of 14.6 months in patients with advanced PanNETs (Table 1). ${ }^{34}$ Addition of everolimus to bevacizumab was associated with further reduction of tumor blood flow in functional CT scans.

\section{Ongoing trial: everolimus versus everolimus + bevacizumab}

On the basis of previous results, the Cancer and Leukemia Group B (CALGB) 80701 trial will evaluate everolimus or everolimus plus bevacizumab in patients with advanced PanNETs, using a randomized phase II design (Table 1).

\section{Expert opinion}

Inhibition of mTOR by everolimus provides significant benefit to patients with PanNETs. Research shows the central role of mTOR is to act as a biological switch. It allows cells to respond to its environment, the availability of nutrients and energy, and a variety of stressors, and it mediates cellular changes that result from interactions with many different growth factors. Upstream of mTOR, information is relayed through complex cascades. This information involves phosphorylation and dephosphorylation of a variety of regulatory molecules, including P13K, Akt, PTEN, Rheb, and TSC. Activation of mTOR modulates the translation of mRNAs that are critical for cell growth and influencing transcription of key genes.

Genetic syndromes involving the mTOR signaling pathway are associated with PanNETs. PI(3)K-Akt-mTOR 
signaling is activated in PanNETs. Studies have shown that there is a link between aberrant PI(3)K-Akt-mTOR and Ras-Raf-MEK-Erk1/2 signaling in PanNETs. Raf kinase inhibitor can suppress Erk 1/2 phosphorylation but strongly induce Akt phosphorylation and VEGF secretion, suggesting the existence of a compensatory feedback loop on PI3KAkt signaling. ${ }^{35}$ Future studies can explore dual targeting of PI(3)K-Akt-mTOR and Ras-Raf-MEK-Erk1/2 signaling in PanNETs. In May 2011, the FDA also approved another targeted agent called sunitinib for PanNETs. Sunitinib (at a dose of $37.5 \mathrm{mg}$ per day) when compared to a placebo showed a doubling in PFS and an increase in objective tumor response. ${ }^{4}$ Both sunitinib and everolimus are effective at improving disease-free survival. Future clinical trials should further investigate these 2 agents in combination or in a sequential manner for the treatment of PanNETs.

Recent studies found rapamycin could concurrently increase the phosphorylation of both Akt and elF-4E, ${ }^{36-38}$ which may counteract with the anticancer efficacy of mTOR inhibitors. These trials suggested that combination therapy with PI3 K-inhibitor ${ }^{36}$ or IGF-1R-inhibitor ${ }^{37,38}$ may overcome the resistance to $\mathrm{mTOR}$-targeted cancer therapy.

Everolimus is mainly an mTORC1 inhibitor. Agents with mTORC2 inhibiting activity could be the study of interest in the future. A recent study showed rapamycin synergized with HSP90 inhibitor enhanced oxidative stress and induced tumor regression in mouse models. ${ }^{39}$ Somatostatin receptor-targeted radionuclide therapy is still investigational and can be considered in combination with everolimus in future studies. Cytotoxic chemotherapy with higher tumor partial response rate may be used in selective patients who require rapid cytoreduction for surgery. Everolimus can be studied in this setting in combination with streptozocin and temozolomide-based chemotherapy in appropriate patients. Finding predictive biomarkers for clinical response to everolimus should be done for individualized therapy.

\section{Acknowledgments}

The authors would like to thank Mika Stepankiw for her editorial assistance.

\section{Disclosure}

The authors report no conflicts of interest in this work

\section{References}

1. Yao JC, Eisner MP, Leary C, et al. Population-based study of islet cell carcinoma. Ann Surg Oncol. 2007;14(12):3492-3500.
2. Yao JC, Hassan M, Phan A, et al. One hundred years after "carcinoid": epidemiology of and prognostic factors for neuroendocrine tumors in 35,825 cases in the United States. J Clin Oncol. 2008;26(18): 3063-3072.

3. Rinke A, Muller HH, Schade-Brittinger C, et al. Placebo-controlled, double-blind, prospective, randomized study on the effect of octreotide LAR in the control of tumor growth in patients with metastatic neuroendocrine midgut tumors: a report from the PROMID Study Group. J Clin Oncol. 2009;27(28):4656-4663.

4. Moertel CG, Lefkopoulo M, Lipsitz S, Hahn RG, Klaassen D. Streptozocin-doxorubicin, streptozocin-fluorouracil or chlorozotocin in the treatment of advanced islet-cell carcinoma. $N$ Engl J Med. 1992; 326(8):519-523.

5. Kouvaraki MA, Ajani JA, Hoff P, et al. Fluorouracil, doxorubicin, and streptozocin in the treatment of patients with locally advanced and metastatic pancreatic endocrine carcinomas. J Clin Oncol. 2004; 22(23):4762-4771.

6. Ekeblad S, Sundin A, Janson ET, et al. Temozolomide as monotherapy is effective in treatment of advanced malignant neuroendocrine tumors. Clin Cancer Res. 2007;13(10):2986-2991.

7. Kulke MH, Stuart K, Enzinger PC, et al. Phase II study of temozolomide and thalidomide in patients with metastatic neuroendocrine tumors. J Clin Oncol. 2006;24(3):401-406.

8. Kulke MH, Hornick JL, Frauenhoffer C, et al. O6-methylguanine DNA methyltransferase deficiency and response to temozolomide-based therapy in patients with neuroendocrine tumors. Clin Cancer Res. 2009; 15(1):338-345.

9. Strosberg JR, Fine RL, Choi J, et al. First-line chemotherapy with capecitabine and temozolomide in patients with metastatic pancreatic endocrine carcinomas. Cancer. 2010;117(2):268-275.

10. Duerr EM, Chung DC. Molecular genetics of neuroendocrine tumors. Best Pract Res Clin Endocrinol Metab 2007;21(1):1-14.

11. Jiao Y, Shi C, Edil BH, et al. DAXX/ATRX, MEN1, and mTOR pathway genes are frequently altered in pancreatic neuroendocrine tumors. Science. 2011;331(6021):1199-1203.

12. Missiaglia E, Dalai I, Barbi S, et al. Pancreatic endocrine tumors: expression profiling evidences a role for AKT-mTOR pathway. J Clin Oncol. 2010;28(2):245-255.

13. Yao JC, Shah MH, Ito T, et al. Everolimus for advanced pancreatic neuroendocrine tumors. N Engl J Med. 2011;364(6):514-523.

14. Crespo JL, Hall MN. Elucidating TOR signaling and rapamycin action: lessons from Saccharomyces cerevisiae. Microbiol Mol Biol Rev. 2002; 66(4):579-591.

15. Dancey JE. Inhibitors of the mammalian target of rapamycin. Expert Opin Investig Drugs. 2005;14(3):313-328.

16. Bjornsti MA, Houghton PJ. The TOR pathway: a target for cancer therapy. Nat Rev Cancer. 2004;4(5):335-348.

17. Beuvink I, Boulay A, Fumagalli S, et al. The mTOR inhibitor RAD001 sensitizes tumor cells to DNA-damaged induced apoptosis through inhibition of p21 translation. Cell. 2005;120(6):747-759.

18. Powis G, Kirkpatrick L. Hypoxia inducible factor-1alpha as a cancer drug target. Mol Cancer Ther. 2004;3(5):647-654.

19. Vaupel P. The role of hypoxia-induced factors in tumor progression. Oncologist. 2004;9 Suppl 5:10-17.

20. Bos JL. Ras oncogenes in human cancer: a review. Cancer Res. 1989; 49(17):4682-4689.

21. Kodaki T, Woscholski R, Hallberg B, Rodriguez-Viciana P, Downward J, Parker PJ. The activation of phosphatidylinositol 3-kinase by Ras. Curr Biol. 1994;4(9):798-806.

22. Nicholson KM, Anderson NG. The protein kinase B/Akt signalling pathway in human malignancy. Cell Signal. 2002;14(5):381-395.

23. deGraffenried LA, Friedrichs WE, Russell DH, Donzis EJ, Middleton AK, Silva JM, et al. Inhibition of mTOR activity restores tamoxifen response in breast cancer cells with aberrant Akt Activity. Clin Cancer Res. 2004;10(23):8059-8067.

24. Sansal I, Sellers WR. The biology and clinical relevance of the PTEN tumor suppressor pathway. J Clin Oncol. 2004;22(14):2954-2963. 
25. Jensen RT, Berna MJ, Bingham DB, Norton JA. Inherited pancreatic endocrine tumor syndromes: advances in molecular pathogenesis, diagnosis, management, and controversies. Cancer. 2008;113(7 Suppl): 1807-1843.

26. Dworakowska D, Grossman AB. Are neuroendocrine tumours a feature of tuberous sclerosis? A systematic review. Endocr Relat Cancer. 2009; 16(1):45-58.

27. Yao JC. Neuroendocrine tumors. Molecular targeted therapy for carcinoid and islet-cell carcinoma. Best Pract Res Clin Endocrinol Metab. 2007;21(1):163-172.

28. von Wichert G, Jehle PM, Hoeflich A, et al. Insulin-like growth factor-I is an autocrine regulator of chromogranin A secretion and growth in human neuroendocrine tumor cells. Cancer Res. 2000;60(16): 4573-4581.

29. Theodoropoulou M, Zhang J, Laupheimer S, et al. Octreotide, a somatostatin analogue, mediates its antiproliferative action in pituitary tumor cells by altering phosphatidylinositol 3-kinase signaling and inducing Zac1 expression. Cancer Res. 2006;66(3):1576-1582.

30. Moreno A, Akcakanat A, Munsell MF, Soni A, Yao JC, Meric-Bernstam F. Antitumor activity of rapamycin and octreotide as single agents or in combination in neuroendocrine tumors. Endocr Relat Cancer. 2008; 15(1):257-266.

31. Zitzmann K, De Toni EN, Brand S, et al. The novel mTOR inhibitor RAD001 (everolimus) induces antiproliferative effects in human pancreatic neuroendocrine tumor cells. Neuroendocrinology. 2007; 85(1):54-60.

32. Yao JC, Phan AT, Chang DZ, et al. Efficacy of RAD001 (everolimus) and octreotide LAR in advanced low- to intermediate-grade neuroendocrine tumors: results of a phase II study. J Clin Oncol. 2008; 26(26):4311-4318.
33. Yao JC, Lombard-Bohas C, Baudin E, et al. Daily oral everolimus activity in patients with metastatic pancreatic neuroendocrine tumors after failure of cytotoxic chemotherapy: a phase II trial. J Clin Oncol. 2010;28(1):69-76.

34. Yao JP, Fogleman AT, Ng D, et al. Randomized run-in study of bevacizumab (B) and everolimus (E) in low- to intermediate-grade neuroendocrine tumors (LGNETs) using perfusion CT as functional biomarker. ASCO 2010. J Clin Oncol. 2010;28:15s(suppl; abstr 4002).

35. Zitzmann K, Ruden J, Brand S, et al. Compensatory activation of Akt in response to $\mathrm{mTOR}$ and Raf inhibitors - a rationale for dual-targeted therapy approaches in neuroendocrine tumor disease. Cancer Lett. 2010; 295(1):100-109.

36. Sun S, Rosenberg LM, Wang X, et al. Activation of Akt and eIF4E survival pathways by rapamycin-mediated mammalian target of rapamycin inhibition. Cancer Res. 2005;65(16):7052-7058.

37. O'Reilly KE, Rojo F, She QB, et al. mTOR inhibition induces upstream receptor tyrosine kinase signaling and activates Akt. Cancer Res. 2006; 66(3):1500-1508

38. Wan X, Harkavy B, Shen N, Grohar P, Helman LJ. Rapamycin induces feedback activation of Akt signaling through an IGF-1R-dependent mechanismFeedback activation of Akt by rapamycin. Oncogene. 2007; 26(13):1932-1940.

39. De Raedt T, Walton Z, Yecies JL, et al. Exploiting cancer cell vulnerabilities to develop a combination therapy for RAS-driven tumors. Cancer Cell. 2011;20(3):400-413.
OncoTargets and Therapy

\section{Publish your work in this journal}

OncoTargets and Therapy is an international, peer-reviewed, open access journal focusing on the pathological basis of all cancers, potential targets for therapy and treatment protocols employed to improve the management of cancer patients. The journal also focuses on the impact of management programs and new therapeutic agents and protocols on

\section{Dovepress}

patient perspectives such as quality of life, adherence and satisfaction The manuscript management system is completely online and includes a very quick and fair peer-review system, which is all easy to use. Visit http://www.dovepress.com/testimonials.php to read real quotes from published authors. 